\title{
Why do Hydrogen and Helium Migrate from Some Planets and Smaller Objects?
}

\author{
Author Weitter Duckss \\ Independent Researcher, Zadar, Croatia \\ mail: wduckss@gmail.com \\ Project: https://www.svemir-ipaksevrti.com/
}

\begin{abstract}
This article analyzes the processes through measuring the material incoming from the outer space onto Earth, through migrating of hydrogen and helium from our atmosphere and from other objects and through inability to detect the radioactive effects on stars and objects with melted interiorities. Habitable periods on such objects are determined through the processes.
\end{abstract}

\section{Introduction}

The goal of the article is to give arguments, based on the existing data bases, that a constant growth of space objects, as well as their rotation and tidal forces, cause their warming up and radiation emissions, therefore making radioactive processes of fission and fusion - which are not detected on stars and other objects anyway - unnecessary. The article gives evidence of hydrogen and helium migrating towards the objects that have more mass and of temperature levels of stars being directly related to their chemical compositions and the objects in their orbits. The argumentation to support a habitable period will be derived from the natural processes of constant growth and matter gathering.

\section{Why there is no radioactive emission, derived from the processes of fission and fusion, inside stars?}

All data bases indicate that astronomic research (or, evidence) support the existence of a constant (monotonous), omnipresent, slow gathering of matter. The processes are "more accelerated" in such part of the Universe where there is more matter gathered (in the form of nebulae, molecular clouds, etc.) during a long period of time, but gathering takes place constantly in the whole volume of the Universe as well. The undisputed evidence of omnipresent gathering are millions of craters on the planets and smaller objects in our system. That process is further supported by the space material incoming daily from the outer space to our planet, with its quantity estimates ranging from 50 [1] to 300 [2] tons per day. Collision and merger of smaller and bigger objects, stars, galaxies [3] and the clusters of galaxies [4] is omnipresent in the whole volume of the Universe.

The processes related with an object's mass and rotation are realized inside the constant process of gathering. [5] Every object and system rotate around their respective axes, except the tidally locked objects. 
When an object has gathered a certain quantity of mass and if there is an adequate speed of rotation, it starts emitting radiation. That happens with the objects, which masses are smaller than the mass of Neptune (Neptune emits about 2,61 times more energy than it receives from the Sun), depending on the speed of rotation around their respective axes (HD $192310 \mathrm{c}$, HD $10180 \mathrm{~g}$, OGLE-2005-BLG-169Lb, OGLE-2017-BLG1434L b, BD-08 $2823 \mathrm{~b}$ ). When an object is influenced by the strong tidal forces, along with its mass and the rotation around the axis, the object melts its interiority ( $Y$ brown dwarfs, Earth, Venus) at the masses that are smaller than those of Earth or Venus (Kepler-70b 0,44 M Earth, temperature $7.662^{\circ} \mathrm{K}$, semi major axis $\left.0,006 \mathrm{AU}\right)$. When an object's rotation speed increases, it decreases the quantity of mass needed for the object to start emitting more radiation than it receives from its main star, i.e., to start creating its own internal processes that result with radiation. If rotation is slower, it takes more mass. It should never be neglected that the outer space has its own temperature, which is higher near a star, and it gets lower (except the thermal deviation; Sun 1 - 5,2 AU [6]).

Radiation and light are not the same thing. There is no light and it is very cold just outside the atmosphere of Sun (outside Earth ... or off the surface of an object that has no atmosphere) (the lowest temperature on Mercury is $80^{\circ} \mathrm{K}$ ). There is no light where radiation is minimal (extremely weak). Light appears on the visible matter (nebulae, planets, ... ) when it gets affected by radiation. The stronger the intensity of radiation waves, the more intensive the visible matter radiates.

Sun does not emit gamma radiation, except from sunspots [7], it emits Xradiation, ultraviolet, visible and infrared radiation and radio waves.

The strongest flashes (and gamma radiation within them) can barely be detected in the total of the Sun's radiation ("the solar constant"). The total of radiation emitted from the sunspots is only one tenth of the energy emitted by Sun per second. [8]

The radioactive processes of fission and fusion are supposed to be followed with enormous radioactive radiation and they should be taking place within Sun, in the core with a diameter, which is $20-25 \%$ of the Sun's radius (radius of Sun is $696.342 \mathrm{~km}$ ). [9] Although gamma radiation, emitted from the sunspots, are relatively minor and hidden in the total of the Sun's radiation, they get detected by the instruments, nevertheless. However, these instruments seem to fail to detect radioactive radiation, supposedly emitted by the object, which diameter is about $300.000 \mathrm{~km}$ long. During the period of 4,6 billion of years (the 
officially recognized age of Sun) [9], the radioactive pollution would pollute a star, as there are no obstacles to prevent the dislocation of matter from the Sun's core to its surface to happen. In all examples of the process of warming up, a warmer fluid or plasma migrates from the warmer parts to the colder ones, in the process of equalizing temperatures. Independent of the statements about the core density, no element or compound is able to maintain its solid state at the temperatures that are many times higher than their boiling points (the forces of pressure in the Sun at the depth of $200.000 \mathrm{~km}$ are $0,2 \mathrm{~g} / \mathrm{m}^{3}$ [10]). All hot elements and compounds, gaseous and liquid alike, migrate towards the surface, while the matter, which is cooled down, goes lower into the interiority of a star (the circular process of equalizing temperatures).

It is also necessary to accept the evidence, provided by astronomers, that stars generally are not radioactive, i.e., radioactive pollution is not detected on them, regardless of their type.

The existence of gamma radiation discharges, which are extremely rare events, can easily be explained with the processes that do not require radioactive pollution of stars. These discharges are related to the poles of fast-rotating stars and galaxies (quasars), and, to a much lesser extent, to the flashes of the stars' spots. The similarity of these two processes is obvious. A fast drift (change) of matter from these spots is similar to the influx of matter into cyclones of fast-rotating stars, where a separation of elements takes place. The influx of a star into a cyclone of a quasar or another fast-rotating galaxy creates flashes of gamma (and all other types of) radiation. The discharge amounts are related to the speed of cyclone and the quantity of newly arrived matter to the eye of the cyclone.

Our Earth (also: Venus, Jupiter, Neptune) is a good example to prove that melted matter is not radioactive and the processes of warmer melted matter and gas being dislocated are omnipresent.

Quote: The forces of pressure, rotation and the forces of attraction create high temperatures, create and determine the systems' appearance, determine the size of radius, surface gravity, the force of magnetic field, chemical composition and the color of objects and a star. Larger objects disintegrate complex compounds and atoms into hydrogen and some helium, due to temperatures above the boiling point of elements and compounds. The rest (approximately 1-1,5\%), 
Sun photospheric composition (by mass): $0.77 \%$ oxygen; $0.29 \%$ carbon; Iron 0.16\%; Neon 0.12\%; Nitrogen 0.09\%; $0.07 \%$ silicon; $0.05 \%$ magnesium; Sulfur $0.04 \%$ ) are also less complex atoms. A sum total of an object's mass, the forces of attraction and the speeds of rotation determine the conditions when a small orbiting object turns into a star. The mass of an object and the speed of its rotation determine the limit when an independent object starts emitting radiation (i.e., starts radiating). [5] end quote

\section{The migration of hydrogen and helium}

When comparing the data from data bases about the chemical composition of the atmospheres (and surfaces) of different objects, it is impossible to ignore the specificity (regularity) that is related to the elements, existing in the atmosphere of an object.

The Sun and gaseous planets (gas giants) - as far as their higher layers, which are the ones that can be successfully measured, are concerned are mostly made of hydrogen and helium (atmosphere by volume: Jupiter, $89 \% \pm 2.0 \%$ hydrogen $\left(\mathrm{H}_{2}\right.$ (molecular hydrogen), $10 \% \pm 2.0 \%$ helium $(\mathrm{He})$; Saturn, $96.3 \pm 2.4 \%$ hydrogen $\left(\mathrm{H}_{2}\right), 3.25 \pm 2.4 \%$ helium $(\mathrm{He})$; Uranus, $83 \pm 3 \%$ hydrogen $\left(\mathrm{H}_{2}\right), 15 \pm 3 \%$ helium $(\mathrm{He})$; Neptune, $80 \% \pm 3.2 \%$ of hydrogen $\left(\mathrm{H}_{2}\right), 19 \% \pm 3.2 \%$ helium $(\mathrm{He})$, Sun, $\mathrm{He} 24,85$ $\%, \mathrm{H} \mathrm{73,46 \%}$ (atomic hydrogen). The other objects have almost no hydrogen in their atmospheres and helium is registered only in traces (Titan $\mathrm{H}_{2}$ 0,2\%, Earth $\mathrm{H}_{2}$ 0,53 ppm, Venus has no $\mathrm{H}_{2}$ and in Mercury's atmosphere only in traces, Mars has no hydrogen, neither molecular nor in compounds nor on the surface, Ceres has no atmosphere, Pluto has no $\mathrm{H}_{2} . \mathrm{H}_{2}$ is also lacking on the other smaller objects (Moon, the moons of Jupiter, itd).

It is known that on Earth there are processes that create large quantities of hydrogen through hydrogen-based compounds: $\mathrm{H}_{2} \mathrm{O}, \mathrm{CH}_{4}$, other hydrocarbons (oil, gas), $\mathrm{NH}_{3}$ etc.). These processes also create large quantities of $\mathrm{H}_{2}$ but it is almost lacking from the atmosphere $(0,53 \mathrm{ppm})$. The existence of the large quantities of $\mathrm{H}_{2}$ results in a proportional appearance of helium $(9 / 1 \mathrm{H} / \mathrm{He}$, which is approximately their average ratio for the whole Universe), but there is no helium in the atmosphere of Earth. There are $\sim 1 \%$ of hydrogen and $\sim 1,84 \%$ of helium appearing in the process of natural gas extraction [11]. Despite of large production of hydrogen and helium, and a constant release of these gases into the atmosphere as well, their share in the atmosphere remains unchanged. 
The loss of hydrogen from the atmosphere of Earth is estimated to be 3 $\mathrm{kg} / \mathrm{s}$ and the one of helium $50 \mathrm{~g} / \mathrm{s}$. [12]

It can be concluded from the existence of melted core of Earth, ever higher average temperatures and shortening the duration and extent of the ice ages [13] that the total factors, which influence the temperature, are constantly growing. There are no data to support the rotation acceleration of Earth (scientists are more inclined towards its deceleration). The same goes for the rotation of Sun, although geologists and astronomers believe that the influence of Sun is constantly increasing (Sun increases its light by $10 \%$ every billion of years) [14]. The increase of the pressure forces grows with the increase of mass, which is registered to be a material incoming from the outer space. In the process of the constant growth, it can be determined that the increase of the mass of Earth is significantly larger than its total material losses.

With regards to the distance of an orbiting object from its main object, the level of space temperature around such an object $(\sim$ minimal temperatures) and the rotation of the object, it can be concluded that hydrogen and helium are found in the atmospheres of the objects with a significant quantity of mass (the planets with impressive atmospheres and Sun). The distance from a main object does not stop the migration of hydrogen and helium to the direction of a main object or the closest object with a sufficient quantity of mass. It is concluded from the atmosphere compositions of internal planets and the satellites of gas giants. There are processes of hydrogen production on Titan $(0,2 \%$ in its atmosphere) but it migrates towards Saturn. Smaller quantities of hydrogen-based compounds are registered in the atmosphere of Pluto (methane $0,25 \%$, ethylene $0,0001 \%$, acetylene $0,0003 \%$, etc [15]) which confirms the existence of the process of creating hydrogen, but the mass of Pluto is insufficient to hold hydrogen and helium in its atmosphere, even though the distances from larger objects are very large and the space temperature is very low. Hydrogen and helium migrate towards the heavier objects, independent of the orbital distance, the level of temperature of such an object and the space around it and the rotation speed around its axis. In our system, the interstitial medium is almost pure vacuum.[16] It means that migrations do not go aimlessly into the space, but towards the heavier objects. It can be read from the chemical composition of the atmospheres of the largest planets that they successfully hold hydrogen and helium, independent of the influence of solar wind, the force of magnetic field and the level of temperature. 


\section{A habitable zone}

To understand the process of life creation, one must understand the process of hydrogen migration, thermal deformations [6], the influence of space temperature on the atmosphere, structure and the rotation of an object [5].

Internal planets have no possibility to create water (in significant quantities) if they lack a melted core, very active geological processes and independent rotation around its axis, because hydrogen, created on the objects with the small quantity of mass, constantly migrates towards Sun. In our system, an independent rotation starts a bit outside the orbit of Venus. The appearance of a planet's independent rotation depends on the mass of Sun and that of the planet and the speed of rotation around the axis of the star. Mars is an equally sterile planet in the orbit of Earth, due to the lack of mass. In the orbit of Mars, Earth would be a frozen object, due to the lack of mass and the lesser effect of the tidal forces (binary effect). Outside the region of thermal deformation (in our system, it is behind the asteroid belt), low temperatures do not support the appearance of oxygen, but support the appearance of hydrogen-based compounds, due to the difference in temperatures of space $(<$ minimal temperatures of planets (the temperatures of space are approximate to the minimal temperatures of their distant satellites): Jupiter $-108-161^{\circ} \mathrm{C}$; Saturn $-189^{\circ} \mathrm{C}$; Uranus $-197,2$ to $-216^{\circ} \mathrm{C}$; Neptune -201 to $-218^{\circ} \mathrm{C}$...) and the boiling point of hydrogen, $-252,87^{\circ} \mathrm{C}$ (when talking about the oxygen compounds, there are only $0,0004 \% \pm 0,0004 \% \mathrm{H}_{2} \mathrm{O}$ on Jupiter; Saturn, Uranus and Neptune have water only in traces; Titan lacks oxygen-based compounds; in the thin atmosphere of Pluto there is only $0,05-0,075 \%$ CO (estimated in 2015. [17]) from the binary effect with its moon, Charon. The melting point of oxygen is at $-218,79^{\circ} \mathrm{C}$ and the boiling point at $-182,962^{\circ} \mathrm{C}$. The temperatures on Jupiter (and its satellites) and Saturn with its satellites are above the boiling point of oxygen, which means all of oxygen would be in the atmosphere without a process to remove it from there and crystallize it on the surface, or it would be a part of compounds (mostly water, since hydrogen is the most represented element there and helium is inert). Traces or extremely small quantities of oxygen and its compounds in the area outside the thermal deformation are the indicator there are some minimal processes of oxygen appearance in this zone after all. One of them is $\mathrm{SO}_{2}$ (its melting point is at -72 and its boiling point at $-10^{\circ} \mathrm{C}$ ) on the moon of lo from the tidal forces of Jupiter and Europa. 
For life to appear in the thermal deformation zone, it takes a proper ratio of mass, the influence of tidal forces and the rotation of stars and planets.

An object needs to have more mass than Earth in the orbit of Mars for the conditions of melting down the interiority of the object to appear and for the geological processes to become very active. Although hydrogen would continue migrating towards Sun, a part of it would create compounds with oxygen, carbon, nitrogen, etc. That is, after all, a basic precondition to create life.

Habitable conditions are also possible for an independent object, placed in a space with a low quantity of matter; as a consequence, such an object would have a very slow rotation (these objects are classified as brown dwarfs). Under such conditions, the melting of the object's interiority is a result of the pressure forces (partially of the rotation, too) and a possible binary effect (Pluto - Charon). There are no processes of volatile elements migrating towards another object or aimlessly into space; all elements are held in the atmosphere and on the surface of the object. An object is habitable in the period before it becomes a star (while it still has a crust). (annex 1.)

There are data, which suggest that objects in very distant orbits may realize such levels of temperature that are comparable to those of stars and it can further be concluded that these objects are also habitable in the period when they still have a crust.

\section{Conclusion}

The migration of hydrogen and helium is directed towards the objects containing more mass. The increase of the Earth's mass through the material incoming from the outer space is bigger than the total of all Earth's material losses. Every independent object and an object in the orbit, with an independent rotation around its axis, the object which is inside the region of thermal deformation, in some period of time is habitable. It is the period when such an object has a crust and the melted interiority, the consequences of which are intensive geological processes. An object's temperature is a result of the pressure forces, the object's rotation and tidal forces (binary effect). These inferences are derived from the measurements of stars, Earth and other objects, where there is no radioactivity that is supposed to be a product of the processes of fission and fusion, just as the following table state. 


\section{Annex 1.}

Planets vs stars (temperature and mass)

\begin{tabular}{|c|c|c|c|c|}
\hline \multicolumn{2}{|c|}{ Planet } & Mass of Jupiter & Temperature $\mathrm{K}$ & Distance AU \\
\hline 1. & $\underline{2 M 1207 b}$ & $4(+6 ;-1)$ & $1600 \pm 100$ & 40 \\
\hline 2 & GQ Lupi b & $1-36(\underline{20})$ & $2650 \pm 100$ & 100 \\
\hline 3 & ROXs 42Bb & 9 & $1800-2,600$ & 157 \\
\hline 4 & HD 106906 b & 11 & 1800 & $\sim 650$ \\
\hline 5 & 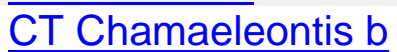 & $10,5-17$ & 2500 & 440 \\
\hline 6 & DH Tauri b & 12 & 2750 & $\underline{330}$ \\
\hline 7 & HD 44627 & $13-14$ & $1600-2400$ & $\overline{275}$ \\
\hline 8 & 2MASS J2126-8140 & $13,3 \pm 1,7$ & 1800 & $6900(>4,500)$ \\
\hline 9 & 1RXS $1609 \mathrm{~b}$ & 14 & 1800 & 330 \\
\hline 10 & UScoCTIO 108 b & 14 & 2600 & 670 \\
\hline 11 & Oph 11 B & 21 & 2478 & 243 \\
\hline 12 & HIP 78530 b & 24 & $2800 \pm 200$ & $710 \pm 60$ \\
\hline
\end{tabular}

\section{Brown Dwarf}

\begin{tabular}{|c|c|c|c|}
\hline 13 & Teide 1 & $57 \pm 15$ & $2600 \pm 150$ \\
\hline 14 & $2 \mathrm{M} \mathrm{J044144}$ & $19 \pm 3 / 9,8 \pm 1,8$ & $2100 / 1800$ \\
\hline 15 & OTS 44 & 11,5 & $1700-2300$ \\
\hline 16 & $\begin{array}{l}\text { DENIS-P J1058.7- } \\
\underline{1548}\end{array}$ & 55 & $1700-2000$ \\
\hline Star & & Mass (Sun 1) & \\
\hline 17 & R Cygni & Cool giant & 2.200 \\
\hline 18 & CW Leonis & $0,7-0,9$ & 2.200 \\
\hline 19 & IK Tauri & 1 & 2.100 \\
\hline 20 & W Aquilae & $1,04-3$ & $\begin{array}{l}1.800(2250 \\
3175)\end{array}$ \\
\hline 21 & T Cephei & $1.5-1.8$ & 2.400 \\
\hline 22 & $\overline{\text { S Pegasi }}$ & 1,8 & 2.107 \\
\hline 23 & Chi Cygni & $2,1+1,5-0,7$ & $2.441-2.742$ \\
\hline 24 & R Leporis & $2,5-5$ & $2.245-2.290$ \\
\hline 25 & R Leonis Minoris & 10,18 & 2.648 \\
\hline 26 & S Cassiopeiae & $\begin{array}{l}\text { loss at } 3.5 \times 10-6 \\
\text { MSun per year }\end{array}$ & 1.800 \\
\hline
\end{tabular}

Table: Cold stars in relationship: mass/radius $S u n=1)$. Planets at a great distance from the stars with high temperatures and different mass.

A few more examples cool Stars: RW Lmi $2.470^{\circ} \mathrm{K}$; V Hya $2.160^{\circ} \mathrm{K}$; II Lup $2.000^{\circ} \mathrm{K}$; V Cyg $1.875^{\circ} \mathrm{K}$; LL Pegasi $2.000^{\circ} \mathrm{K}$; LP And $2.040^{\circ} \mathrm{K}$; V384 Per $1.820^{\circ} \mathrm{K} ;$ W Ori $2.625^{\circ} \mathrm{K}$; S Aur $1.940^{\circ} \mathrm{K}$; QZ Mus $2.200^{\circ} \mathrm{K}$; AFGL $42022.200^{\circ} \mathrm{K}$ : V821 Her $2.200^{\circ} \mathrm{K}$; V1417 Aql $2.000^{\circ} \mathrm{K}$; S Cep $2.095^{\circ} \mathrm{K}$; RV Cyg $2.675^{\circ} \mathrm{K}$ etc.

Tables from my article (with minor modifications) [5]

\section{Reference}

[1]. https://solarsystem.nasa.gov/asteroids-comets-and-meteors/meteors-andmeteorites/overview/?page=0\&per page=40\&order=id+asc\&search=\&condition $1=\mathrm{m}$ eteor shower\%3Abody type "What is a Meteor Shower?" 
[2]. https://cordis.europa.eu/project/rcn/102627/reporting/en Cosmic Dust in the Terrestrial Atmosphere

[3]. https://en.wikipedia.org/wiki/Category:Interacting galaxies

[4].

http://www.spacetelescope.org/static/archives/releases/science papers/heic1506a.p df "The non-gravitational interactions of dark matter in colliding galaxy clusters" David Harvey, Richard Massey, Thomas Kitching, Andy Taylor, Eric Tittley

[5]. https://www.svemir-ipaksevrti.com/Universe-and-rotation.html\#Effects-of-

Rotation-Arund-the-Axis-on-the-Stars-Galaxy-and-Rotation-of-Universe

DOI: $10.18483 /$ ijSci.1908 „Effects of Rotation Around the Axis on the Stars, Galaxy and Rotation of Universe" W.Duckss

[6].http://www.sciencepublishinggroup.com/journal/paperinfo?journalid=301\&doi=10. 11648/j.ajaa.20180603.13 "The Processes Which Cause the Appearance of Objects and Systems" W.Duckss

[7]. https://www.nasa.gov/mission pages/GLAST/news/highest-energy.html „NASA's Fermi Detects the Highest-Energy Light From a Solar Flare" June 11, 2012

[8].

https://www.windows2universe.org/?page=/sun/spectrum/multispectral sun overvie w.html "The Multispectral Sun"

[9]. http://science.sciencemag.org/content/338/6107/651.full "Apsolutna kronologija i termička obrada čvrstih tvari u solarnom protoplanetarnom disku" James N.

Connelly, Martin Bizzarro, Alexander N. Krot, Åke Nordlund, Daniel Wielandt, Marina A. Ivanova

[10].

https://web.archive.org/web/20130510142009/http://mynasa.nasa.gov/worldbook/sun worldbook.html "World Book at NASA“

[11].

https://www.jstor.org/stable/3624173?origin=crossref\&seq=1\#metadata info tab con tents "Composition of Gas from a Well at Dexter, Kan." D. F.

McFarland. Transactions of the Kansas Academy of Science (1903-) Vol. 19 (1903 1904), pp. 60-62 (3 pages). doi : $10.2307 / 3624173$. JSTOR 3624173

[12]. http://www.eltereader.hu/media/2014/04/Atmospheric Chemistry READER.pdf „Atmospheric Chemistry“ István Lagzi; Róbert Mészáros; Györgyi Gelybó; Ádám Leelőssy, Copyright @ 2013 Eötvös Loránd University [13].

https://www.jstor.org/stable/633219?origin=crossref\&seq=1\#page scan tab content s Lockwood, J.G.; van Zinderen-Bakker, E. M. (November 1979). "The Antarctic IceSheet: Regulator of Global Climates?: Review". The Geographical Journal. 145 (3): 469-471. doi:10.2307/633219. JSTOR 633219.

[14]. http://theconversation.com/the-sun-wont-die-for-5-billion-years-so-why-dohumans-have-only-1-billion-years-left-on-earth-37379 "The sun won't die for 5 billion years, so why do humans have only 1 billion years left on Earth? Jillian Scudder" Postdoctoral Research Fellow in Astrophysics, University of Sussex [15]. Gurwell, M.; Lellouch, E.; Butler, B.; et al. (November 2015). "Detection of Atmospheric CO on Pluto with ALMA". American Astronomical Society, DPS meeting \#47, \#105.06. Bibcode:2015DPS....4710506G. 
[16]. http://www.newworldencyclopedia.org/entry/Outer space\#Environment „Environment

Outer space is the closest natural approximation of a perfect vacuum."

[17]. http://adsabs.harvard.edu/abs/2015DPS...4710506G Gurwell, M.; Lellouch, E.; Butler, B.; et al. (November 2015). "Detection of Atmospheric CO on Pluto with ALMA". American Astronomical Society, DPS meeting \#47, \#105.06. Bibcode:2015DPS....4710506G.

Keywords: Migration of hydrogen; Habitable zone; Constant growth of matter; the effects of rotation; 